\title{
Development of Trifluoromethanesulfonic Acid-Immobilized Nitrogen-Doped Carbon-Incarcerated Niobia Nanoparticle Catalysts for Friedel-Crafts Acylation
}

Xi Yang, Tomohiro Yasukawa*, Yasuhiro Yamashita, and ShūKobayashi*

Department of Chemistry, School of Science, The University of Tokyo, Hongo, Bunkyo-ku, Tokyo 113-0033, Japan 


\section{Contents}

\section{Supporting Information}

1. Optimization of NCI-Nb-TfOH ---



3. Friedel-Crafts acylation ------------------------------------------------ S-3

4. STEM analysis and EDS mapping ------------------------------------------------------ S-4

5. FTIR --------------- S-7

6. NMR charts -------

\section{Optimization of preparation procedure of NCI-Nb-TfOH:}

Table S1. Optimization of catalyst preparation
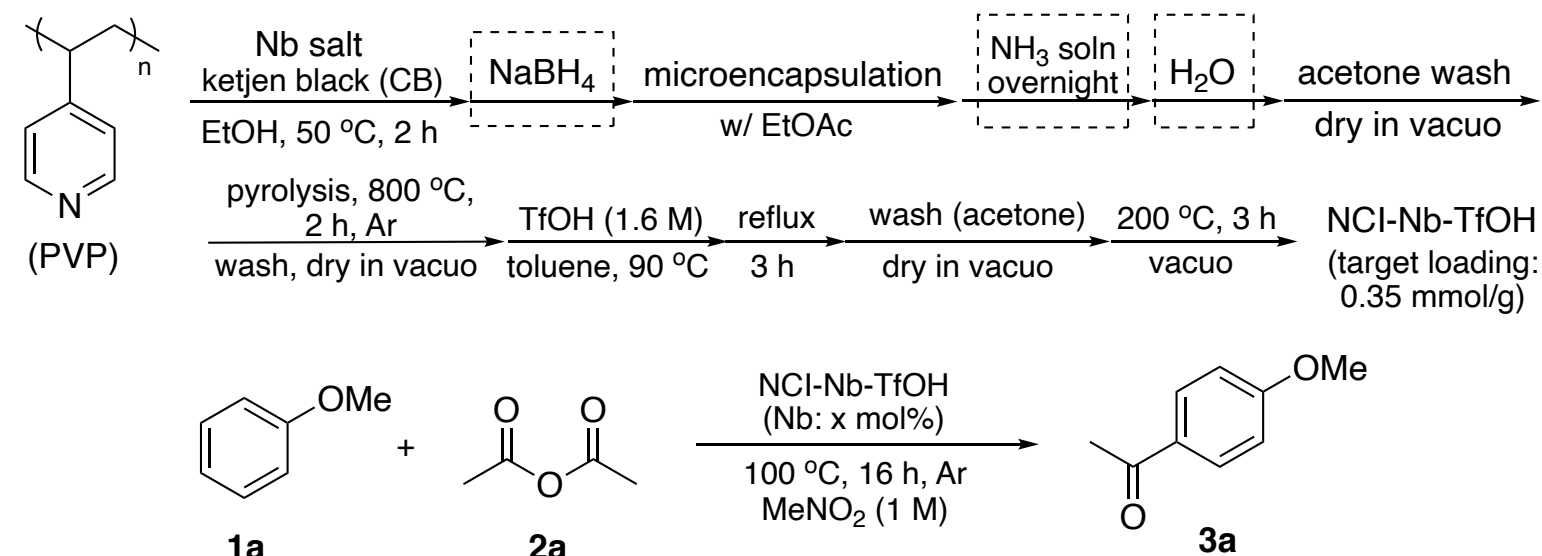

\begin{tabular}{|c|c|c|c|c|c|c|c|c|}
\hline & 1 & & & $2 a$ & & & $3 a$ & \\
\hline entry & $\mathrm{NaBH}_{4}$ & $\mathrm{NH}_{3}$ & $\mathrm{H}_{2} \mathrm{O}$ & PVP:CB & $\mathrm{Nb}$ salt & $\begin{array}{c}\text { metal loading } \\
(\mathrm{mmol} / \mathrm{g})^{\mathrm{a}}\end{array}$ & $\begin{array}{c}\text { cat. loading } \\
(\mathrm{Nb}: \mathrm{x} \text { mol\%) }\end{array}$ & $\begin{array}{l}\text { yield } \\
(\%)^{b}\end{array}$ \\
\hline 1 & \multirow{4}{*}{$\sqrt{ }$} & \multirow{4}{*}{$\sqrt{ }$} & \multirow{4}{*}{$\sqrt{ }$} & \multirow{2}{*}{$1: 1$} & \multirow{2}{*}{$\mathrm{NbCl}_{5}$} & \multirow{2}{*}{0.115} & $2 \mathrm{~mol} \%$ (87 mg) & 84 \\
\hline 2 & & & & & & & $1 \mathrm{~mol} \%$ (43 mg) & 66 \\
\hline 3 & & & & \multirow{2}{*}{$1: 2$} & $\mathrm{NbCl}_{5}$ & 0.063 & $0.5 \mathrm{~mol} \%$ (40 mg) & 57 \\
\hline 4 & & & & & $\mathrm{Nb}(\mathrm{OEt})_{5}$ & 0.043 & $0.5 \mathrm{~mol} \%(58 \mathrm{mg})$ & 69 \\
\hline 5 & $\sqrt{ }$ & $x$ & $\sqrt{ }$ & $1: 2$ & $\mathrm{NbCl}_{5}$ & 0.320 & $2 \mathrm{~mol} \%$ (31 mg) & 51 \\
\hline 6 & $\sqrt{ }$ & $x$ & $x$ & $1: 2$ & $\mathrm{NbCl}_{5}$ & 0.364 & $2 \mathrm{~mol} \%$ (27 mg) & 39 \\
\hline 7 & $x$ & $x$ & $\sqrt{ }$ & $1: 2$ & $\mathrm{NbCl}_{5}$ & 0.106 & $1 \mathrm{~mol} \%$ (47 mg) & 84 \\
\hline 8 & \multirow{3}{*}{$x$} & \multirow{3}{*}{$x$} & \multirow{3}{*}{$x$} & \multirow{3}{*}{$1: 2$} & $\mathrm{NbCl}_{5}$ & 0.337 & $2 \mathrm{~mol} \%$ (30 mg) & 84 \\
\hline $9^{c}$ & & & & & $\mathrm{NbCl}_{5}$ & 0.346 & $2 \mathrm{~mol} \%$ (29 mg) & 71 \\
\hline 10 & & & & & $\mathrm{Nb}(\mathrm{OEt})_{5}$ & 0.361 & $2 \mathrm{~mol} \%$ (28 mg) & 64 \\
\hline 11 & \multirow{3}{*}{$x$} & \multirow{3}{*}{$\sqrt{ }$} & \multirow{3}{*}{$x$} & \multirow{3}{*}{$1: 2$} & $\mathrm{NbCl}_{5}$ & 0.244 & $1 \mathrm{~mol} \%$ (20 mg) & 83 \\
\hline $12^{\mathrm{d}}$ & & & & & $\mathrm{NbCl}_{5}$ & 0.240 & $1 \mathrm{~mol} \%$ (21 mg) & 82 \\
\hline $13^{\mathrm{d}}$ & & & & & $\mathrm{NbCl}_{5}$ & 0.234 & $1 \mathrm{~mol} \%(21 \mathrm{mg})$ & 85 \\
\hline
\end{tabular}

${ }^{a}$ Determined by ICP analysis. ${ }^{b}$ Determined by GC analysis. ${ }^{c}$ Reproduced results of entry $8 .{ }^{\mathrm{d}}$ Reproduced results of entry 11 


\section{Elemental analysis of NCI-Nb-TfOH and NC-TfOH:}

Table S2. Elemental analysis

\begin{tabular}{ccccccc}
\hline catalyst & $\mathrm{C} \%$ & $\mathrm{H} \%$ & $\mathrm{~N} \%$ & $\mathrm{~S} \%$ & $\mathrm{~F} \%$ & $\begin{array}{c}\text { estimated TfOH } \\
(\mathrm{mmol} / \mathrm{g})^{\mathrm{a}}\end{array}$ \\
\hline NCI-Nb-TfOH & 87.04 & 0.76 & 1.82 & 1.17 & 1.83 & 0.321 \\
NC-TfOH & 91.01 & 0.74 & 1.12 & 1.84 & 2.14 & 0.375
\end{tabular}

${ }^{a}$ Determined from the fluorine percentage.

\section{Friedel-Crafts acylation}

\section{3-1. Recovery and reuse:}

Table S3. Recover and reuses of NCI-Nb-TfOH



${ }^{a}$ Determined by GC analysis with nitrobenzene as the internal standard. ${ }^{\mathrm{b}} \mathrm{TfOH}$ treatment of the used catalyst was conducted before reaction following the general procedure.

\section{3-2. TfOH catalyzed Friedel-Crafts acylation:}

Table S4. Examination of TfOH catalyzed Friedel-Crafts acylation

\begin{tabular}{|c|c|c|}
\hline $1 a$ & $\begin{array}{c}\mathrm{TfOH}(\mathrm{x} \mathrm{mol} \%) \\
100^{\circ} \mathrm{C}, 16 \mathrm{~h}, \mathrm{Ar} \\
\mathrm{MeNO}_{2}(1 \mathrm{M})\end{array}$ & 3a \\
\hline entry & $\mathrm{x}(\mathrm{mol} \%)$ & yield $(\%)^{\mathrm{a}}$ \\
\hline 1 & 1.0 & 76 \\
\hline 2 & 0.1 & 40 \\
\hline 3 & 0.01 & trace \\
\hline
\end{tabular}

${ }^{a}$ Determined by GC analysis with nitrobenzene as the internal standard.

\section{3-3. Friedel-Crafts acylation with water as additive:}

Table S5. Examination of water as additive

\begin{tabular}{|c|c|c|c|}
\hline $1 \mathbf{a}$ & $\begin{array}{r}\mathrm{NCl} \\
\text { or } \mathrm{NCl} \\
\text { Additive: } \mathrm{I} \\
100^{\circ} \mathrm{C}\end{array}$ & $\begin{array}{l}2 \text { mol\%) } \\
\mathrm{Nb}: 1 \mathrm{~mol} \%) \\
\% \text { or } 1 \text { equiv } \\
\mathrm{eNO}_{2}(1 \mathrm{M})\end{array}$ & $3 \mathbf{a}$ \\
\hline entry & catalyst & $\mathrm{H}_{2} \mathrm{O}$ & yield $^{2}$ \\
\hline 1 & NCI-Nb-TfOH & - & 85 \\
\hline 2 & (Nb: 1 mol\%) & $10 \mathrm{~mol} \%$ & 83 \\
\hline
\end{tabular}




\begin{tabular}{cccc}
3 & & 1.0 equiv. & 65 \\
4 & NCI-Ti-TfOH & - & 83 \\
5 & $($ Ti: $2 \mathrm{~mol} \%)$ & 10 mol\% & 74 \\
6 & 1.0 equiv, & 57 \\
\hline
\end{tabular}

${ }^{a}$ Determined by GC analysis with nitrobenzene as the internal standard.

\section{STEM and EDS mapping}

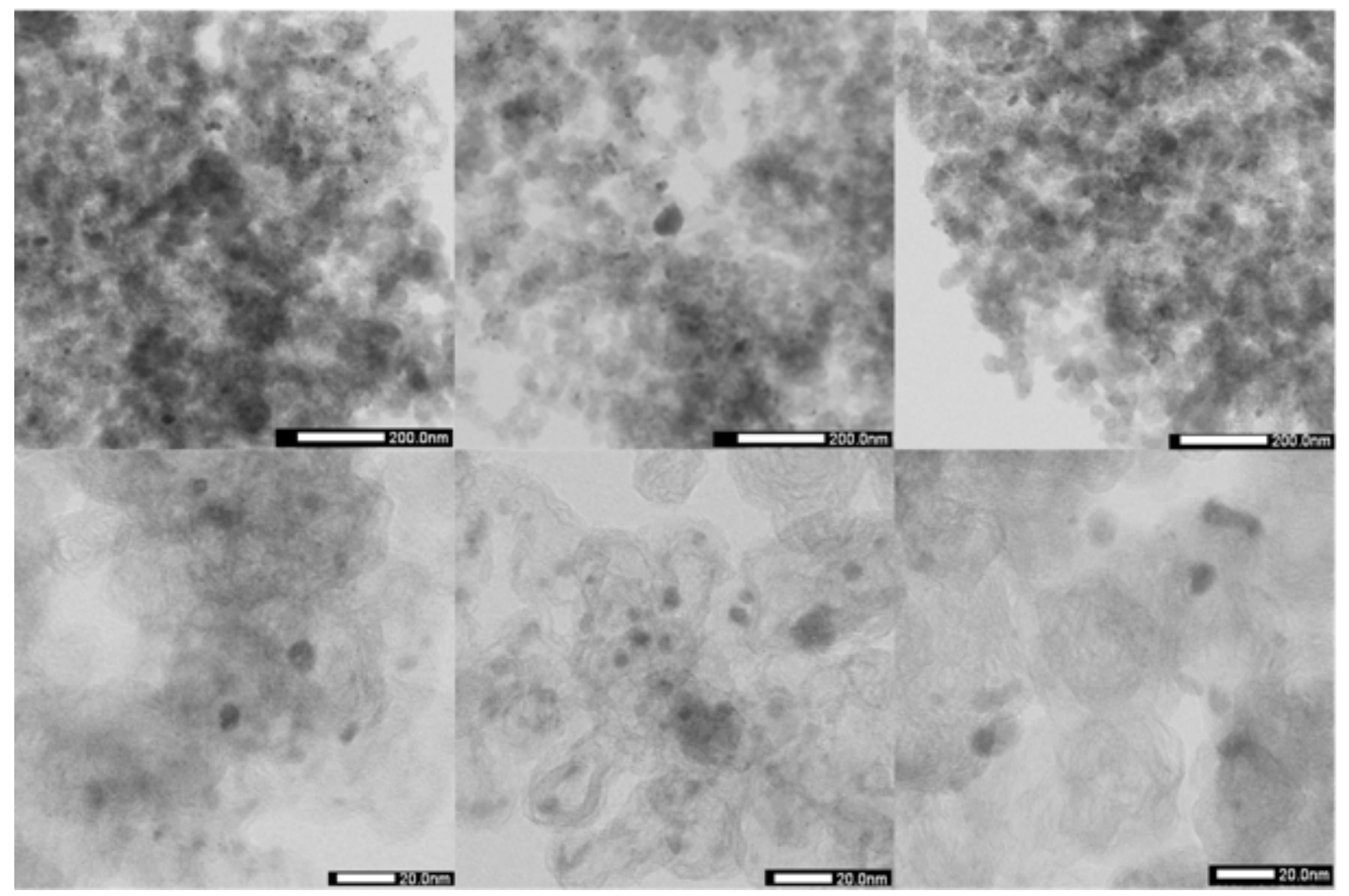

Figure S1. STEM analysis of NCI-Nb-TfOH 




Figure S2. EDS mapping of NCI-Nb-TfOH 

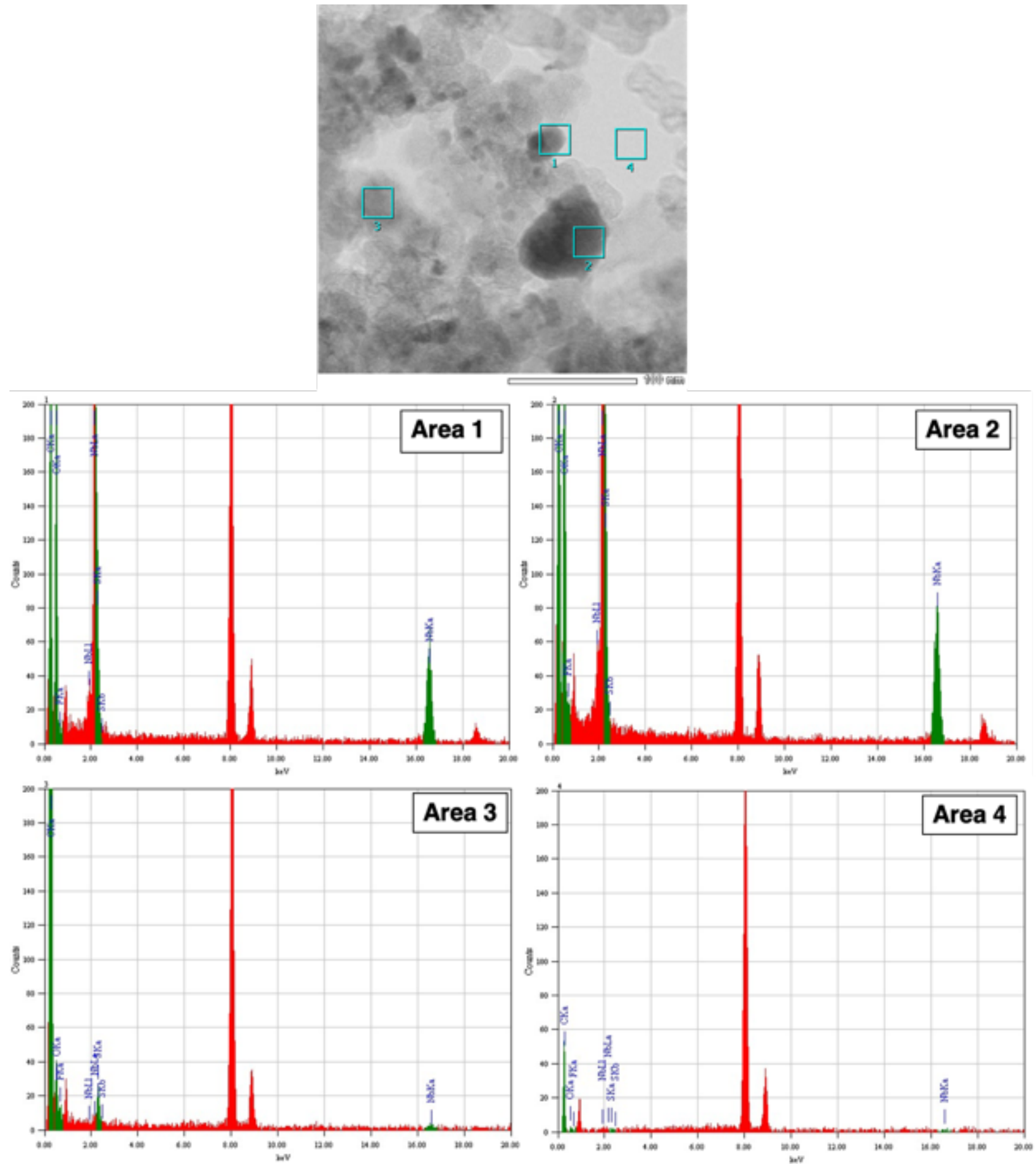

Figure S3. Area analysis of NCI-Nb-TfOH 


\section{FTIR}

5-1. FTIR of NCI-Nb-TfOH



Figure S4. FTIR of NCI-Nb-TfOH

\section{5-2. FTIR of NC-TfOH}

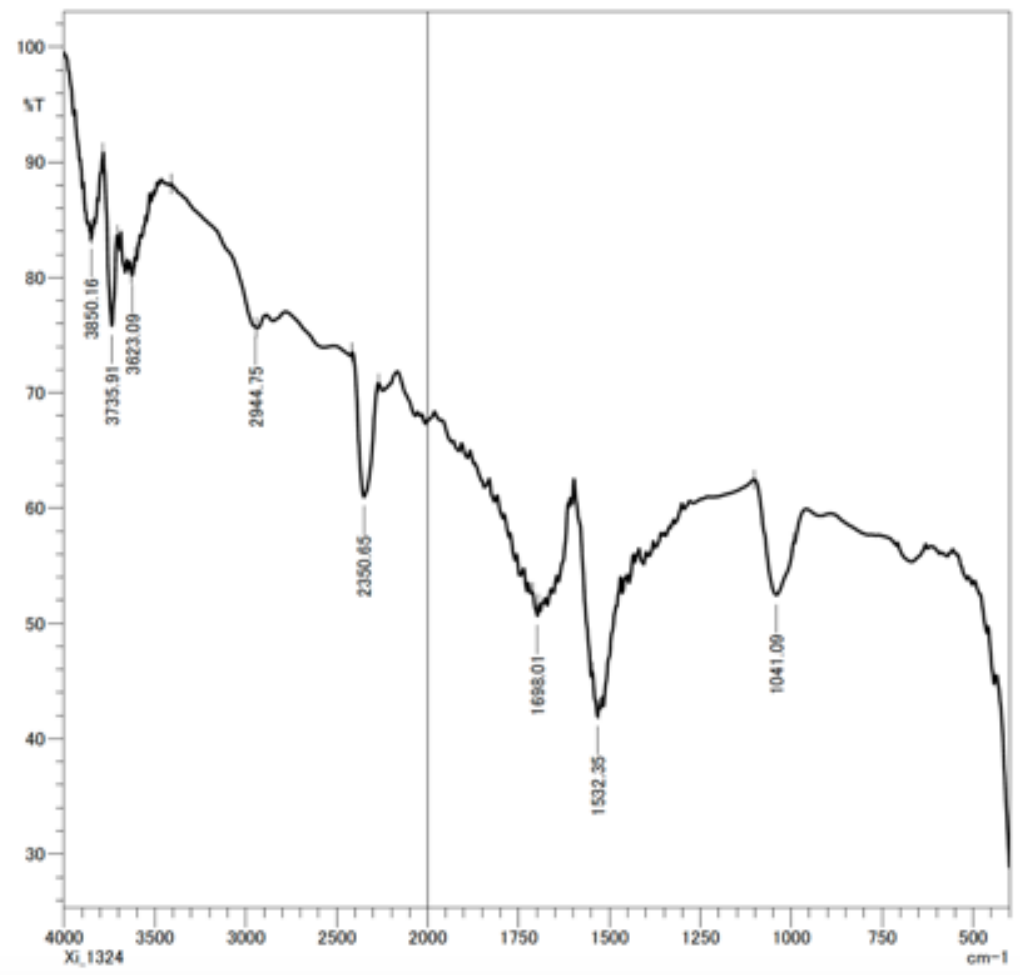

Figure S5. FTIR of NC-TfOH 
5-3. FTIR of pyridine absorption on NCI-Nb-TfOH

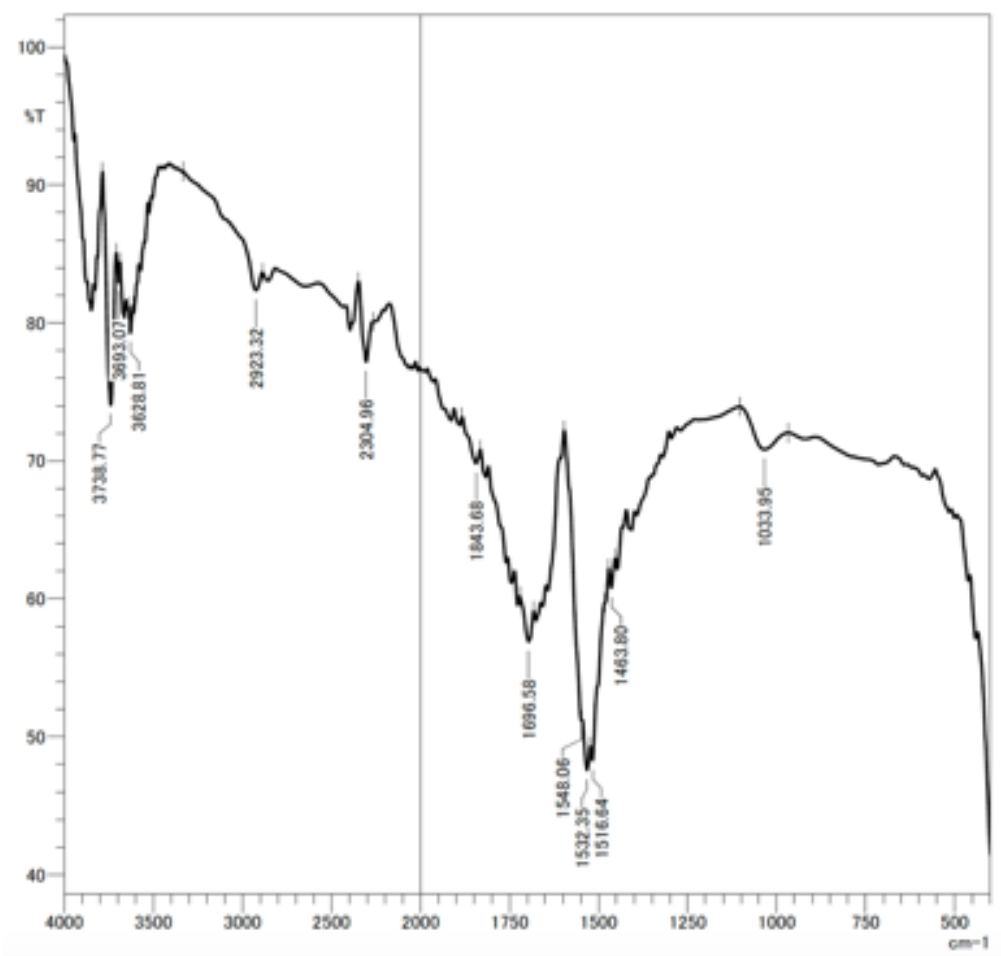

Figure S6. FTIR of pyridine absorption on NCI-Nb-TfOH

\section{5-4. FTIR of pyridine absorption on NC-TfOH}



Figure S7. FTIR of pyridine absorption on NC-TfOH 
6. NMR

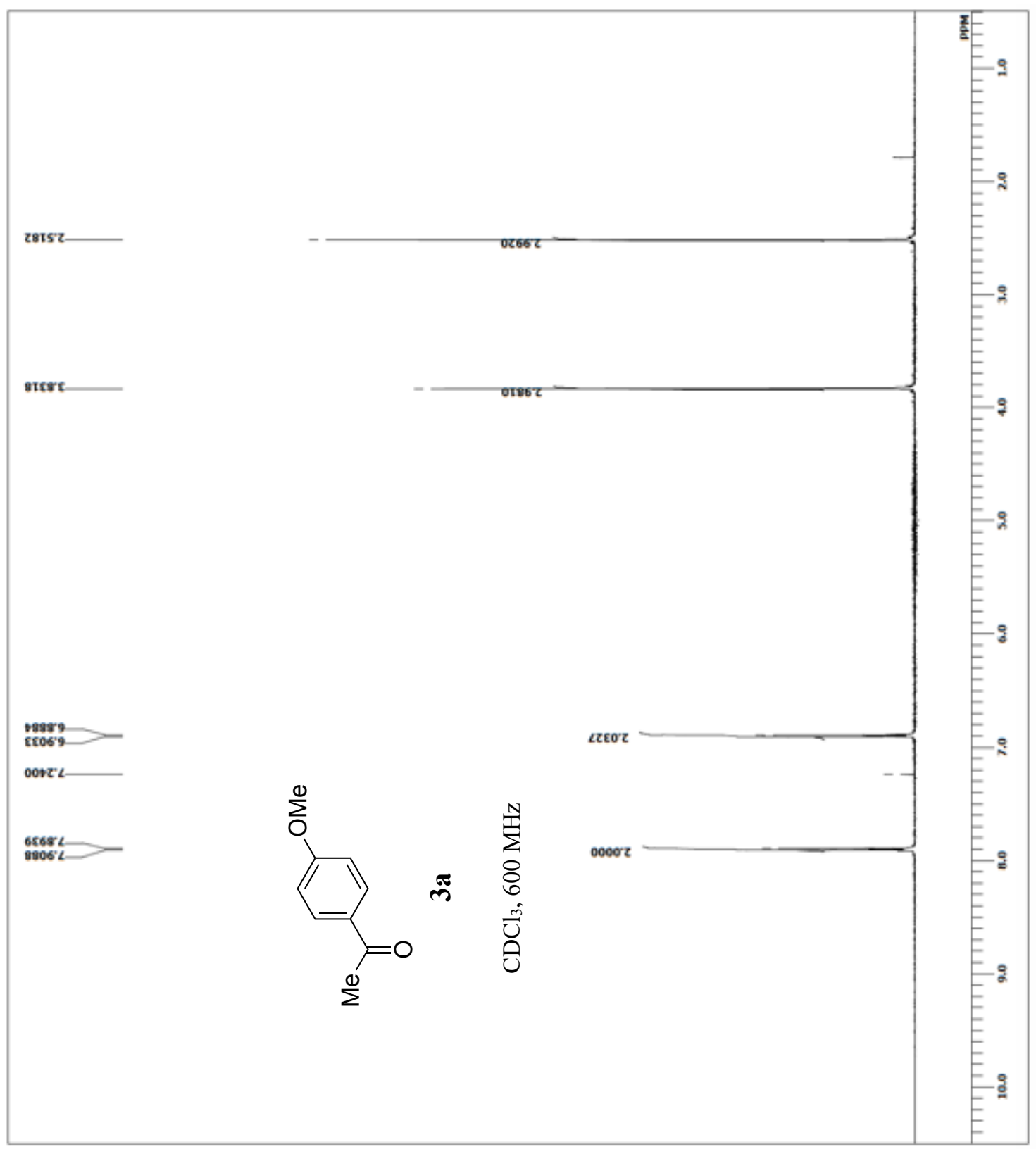




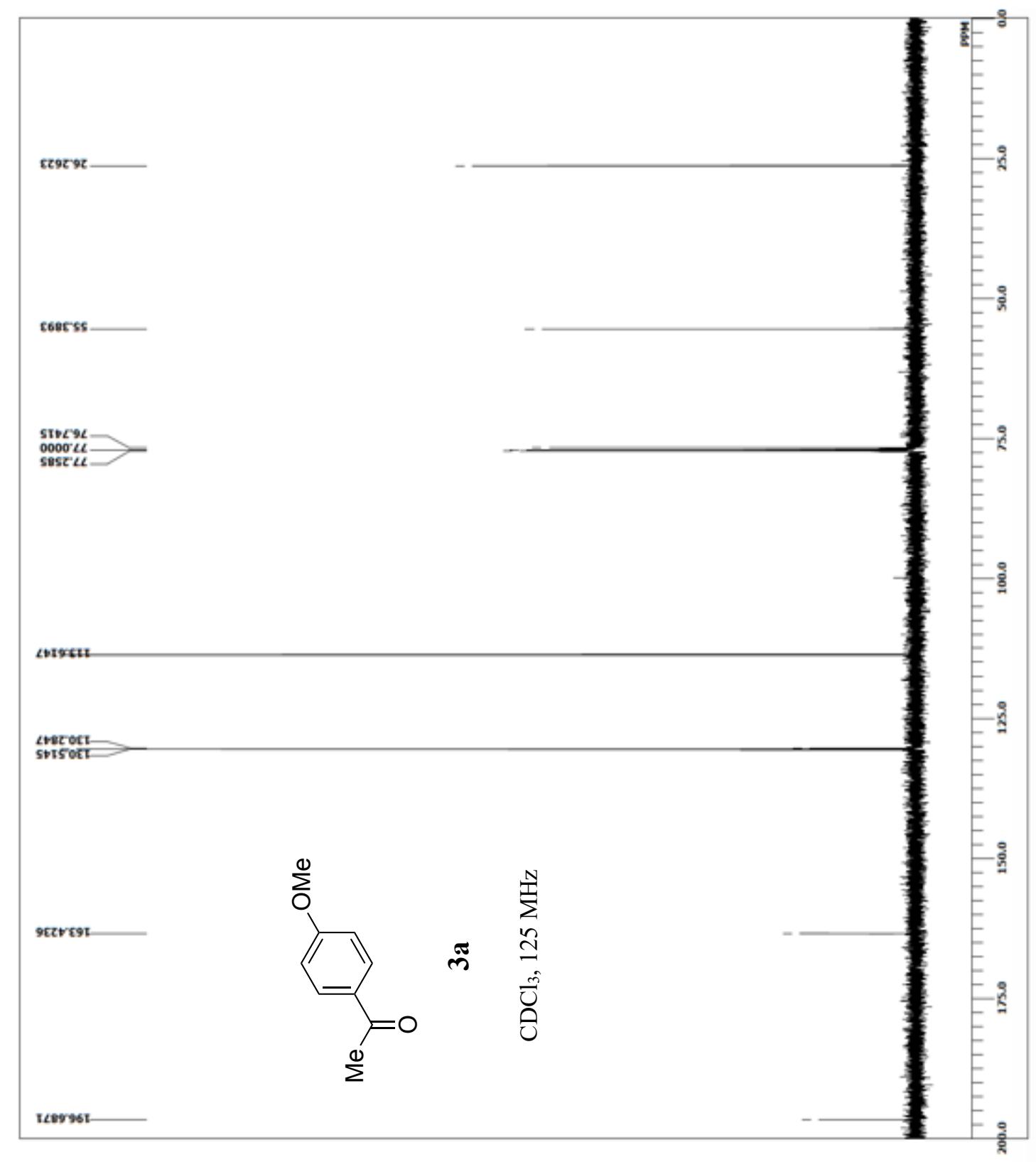






S-11 


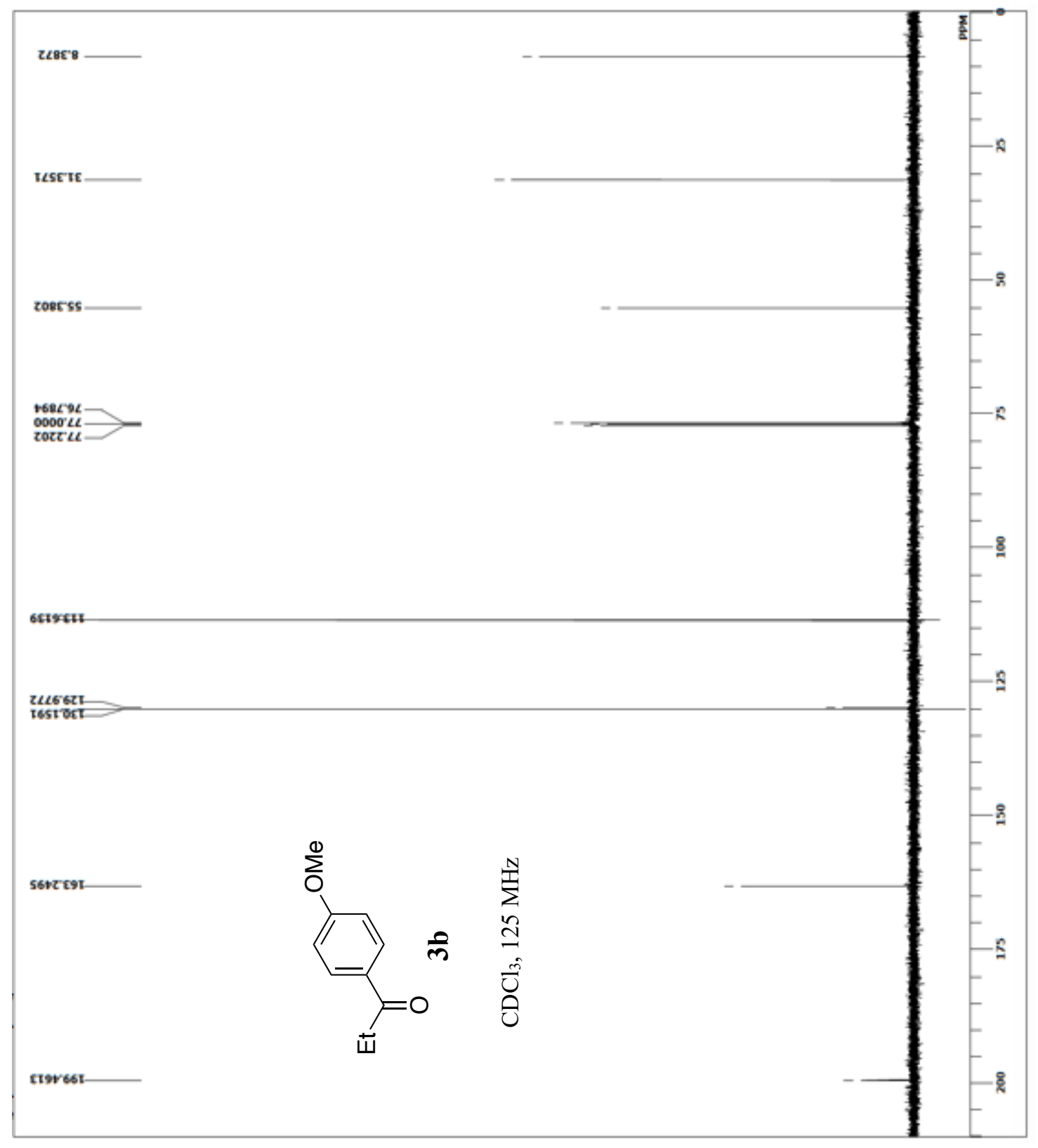




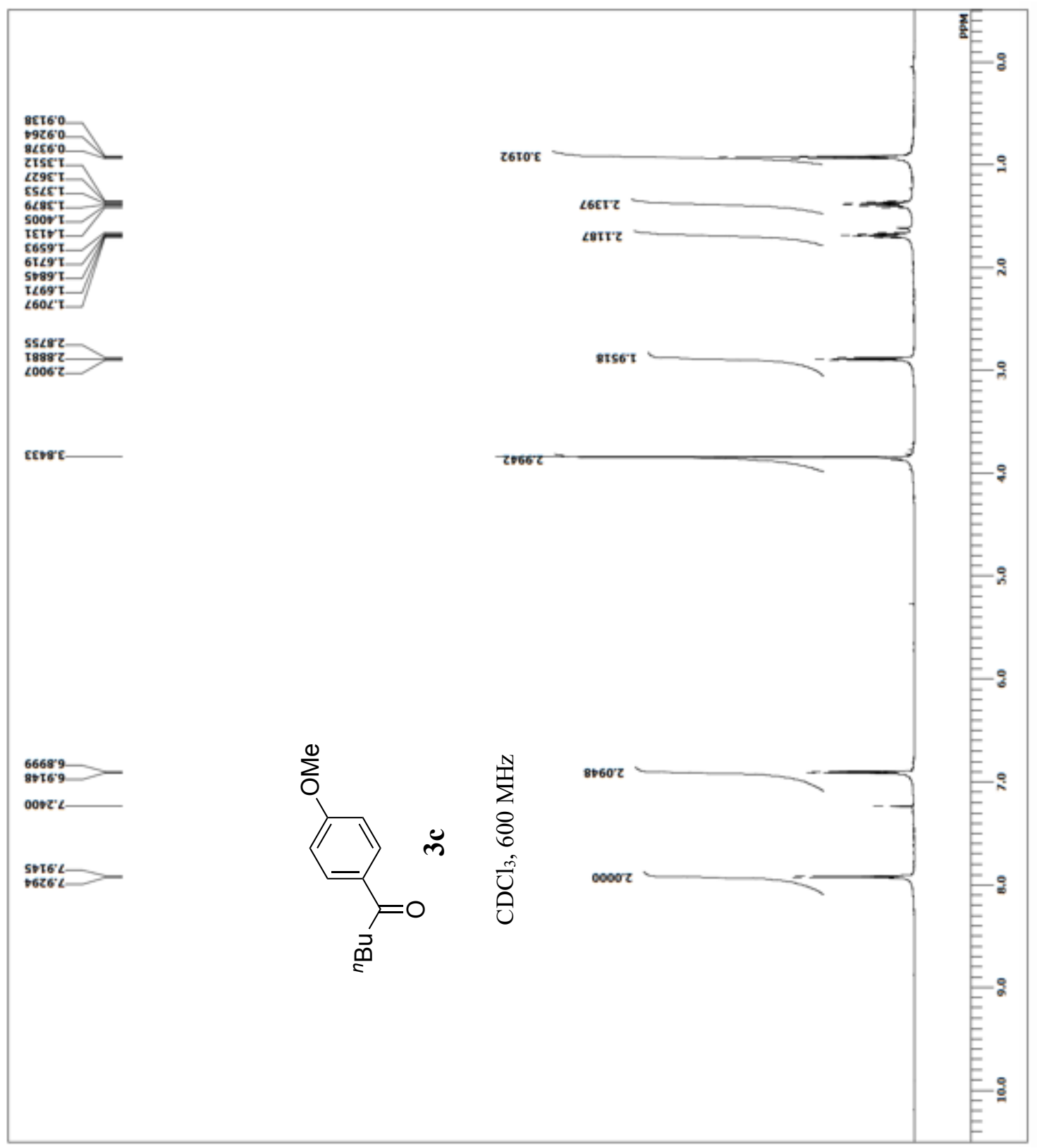




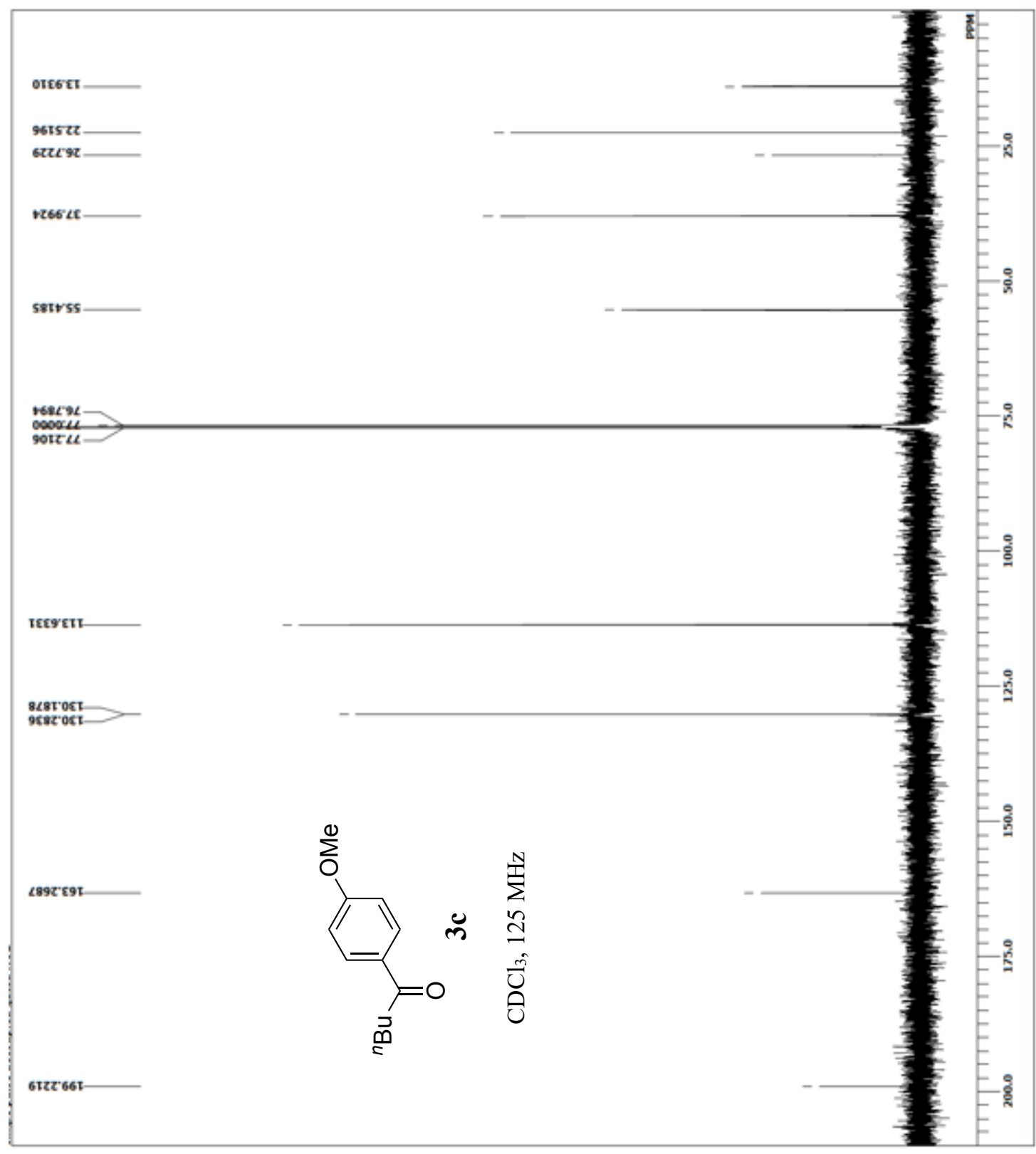




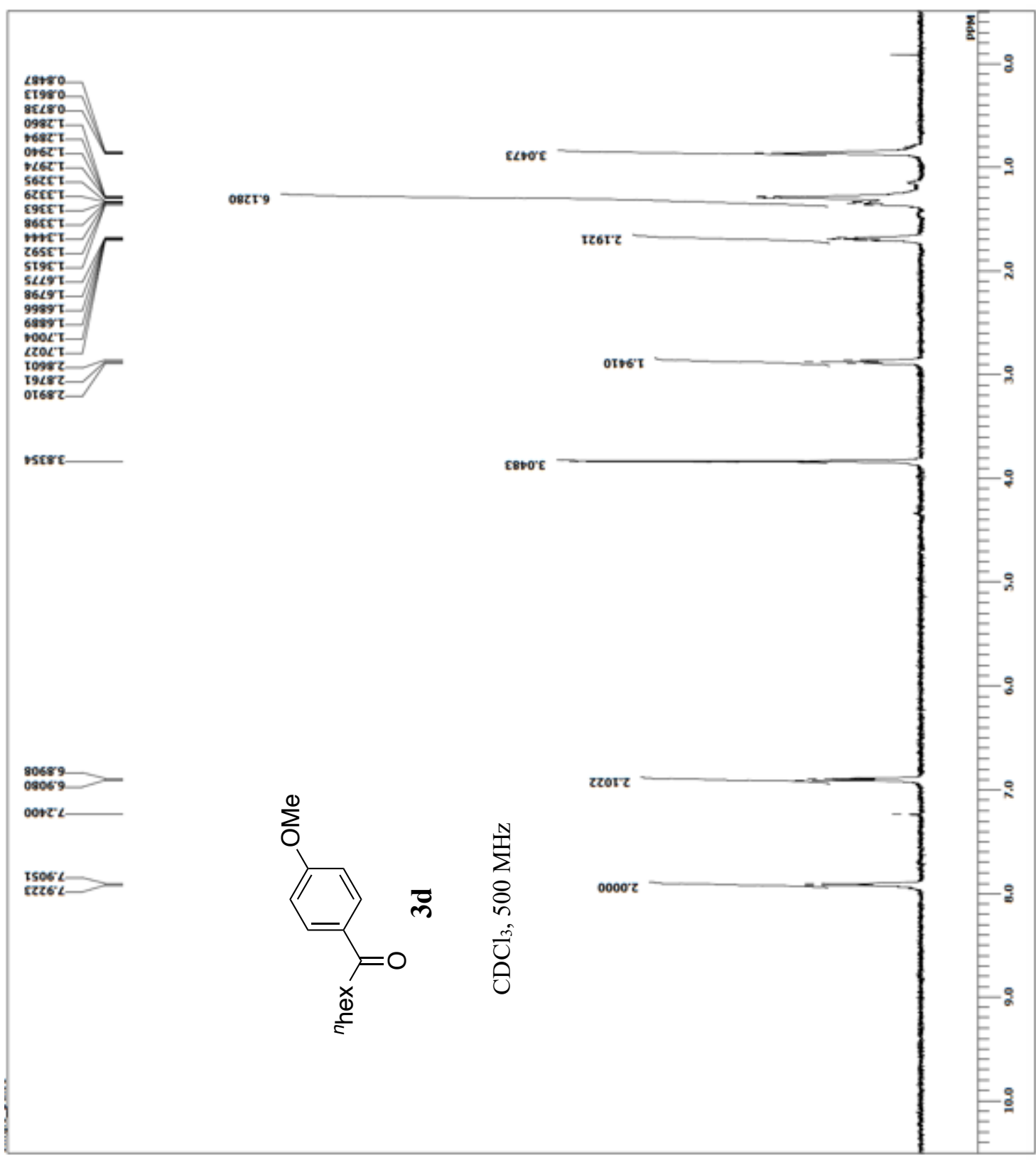




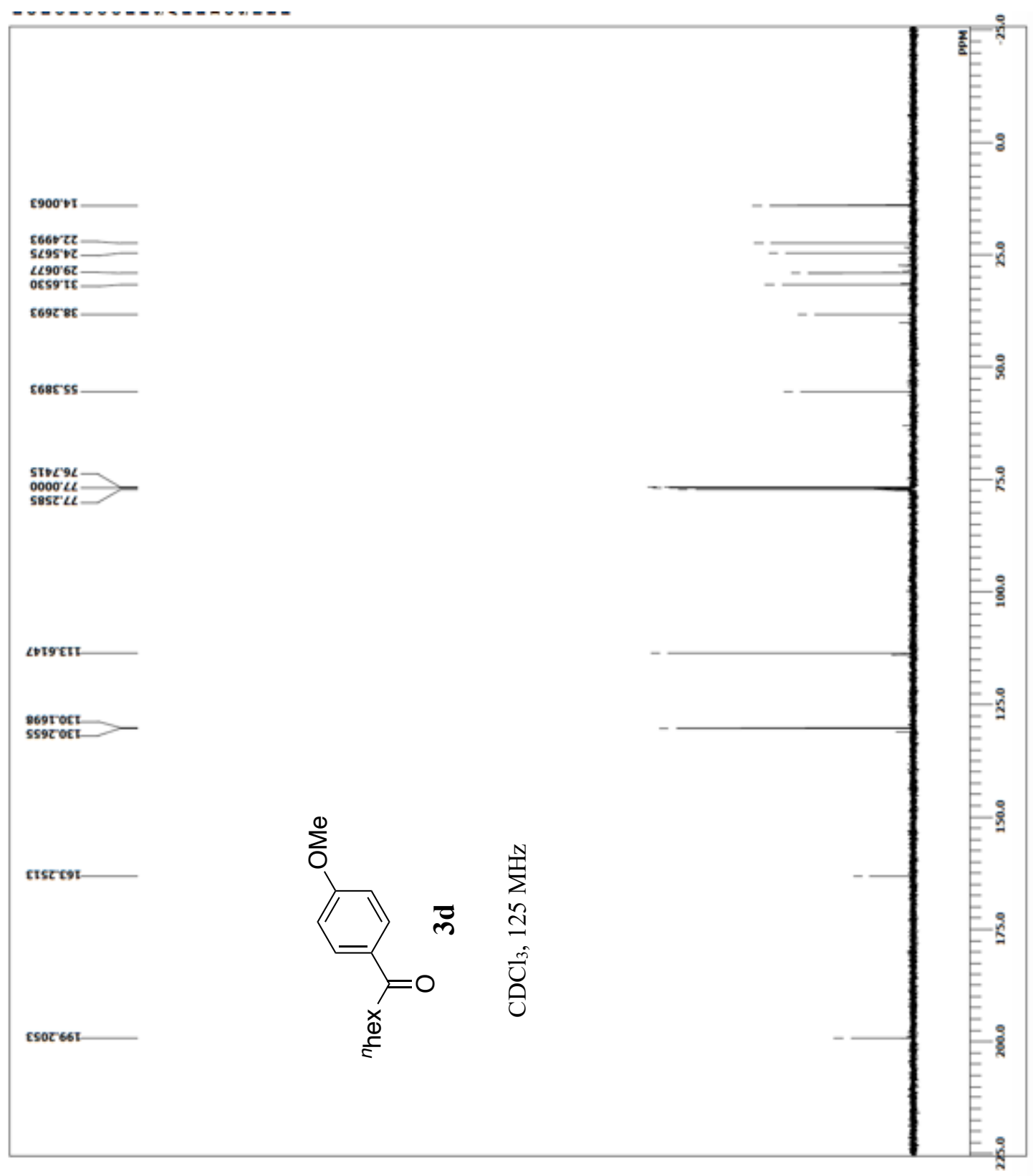









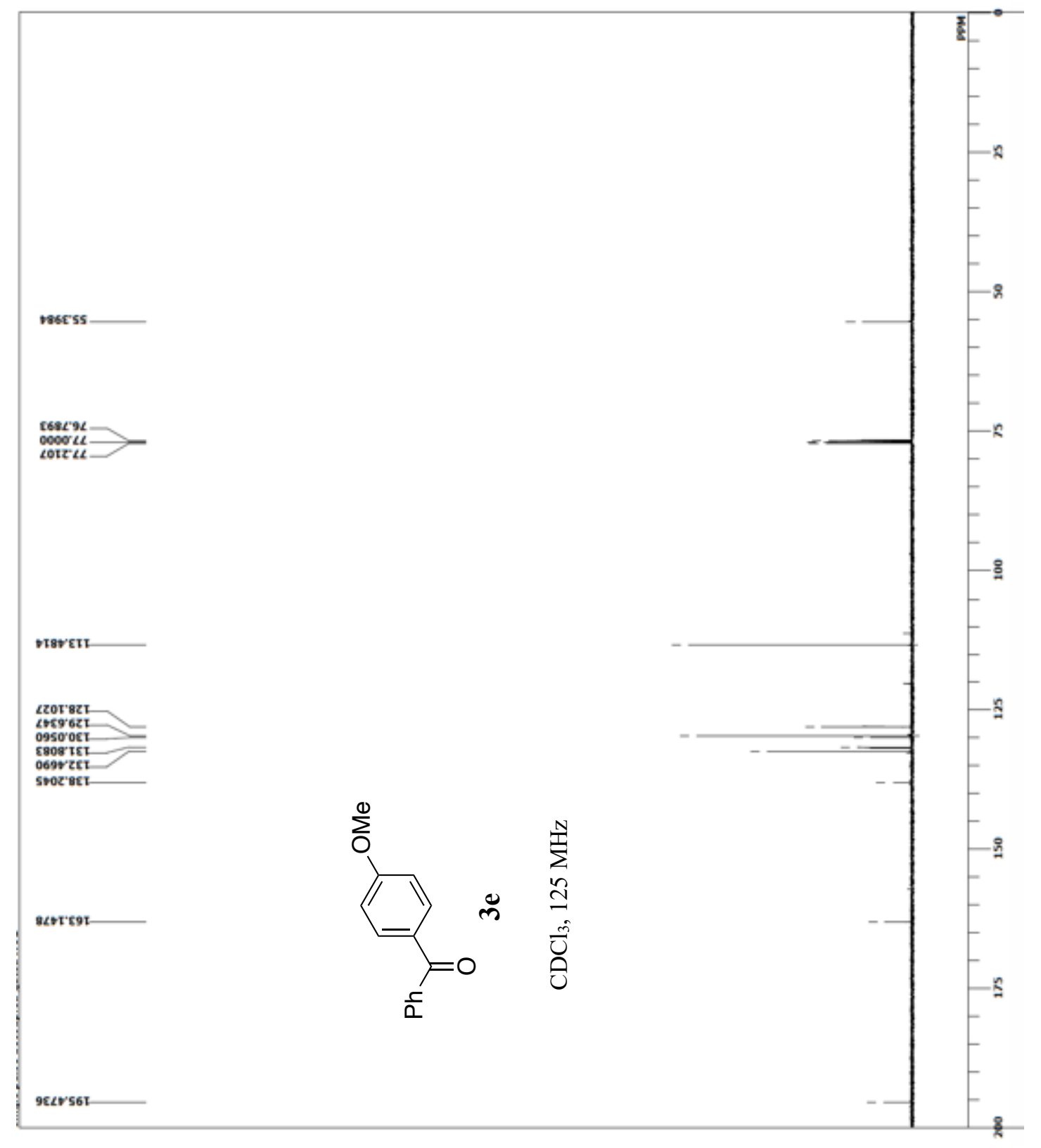




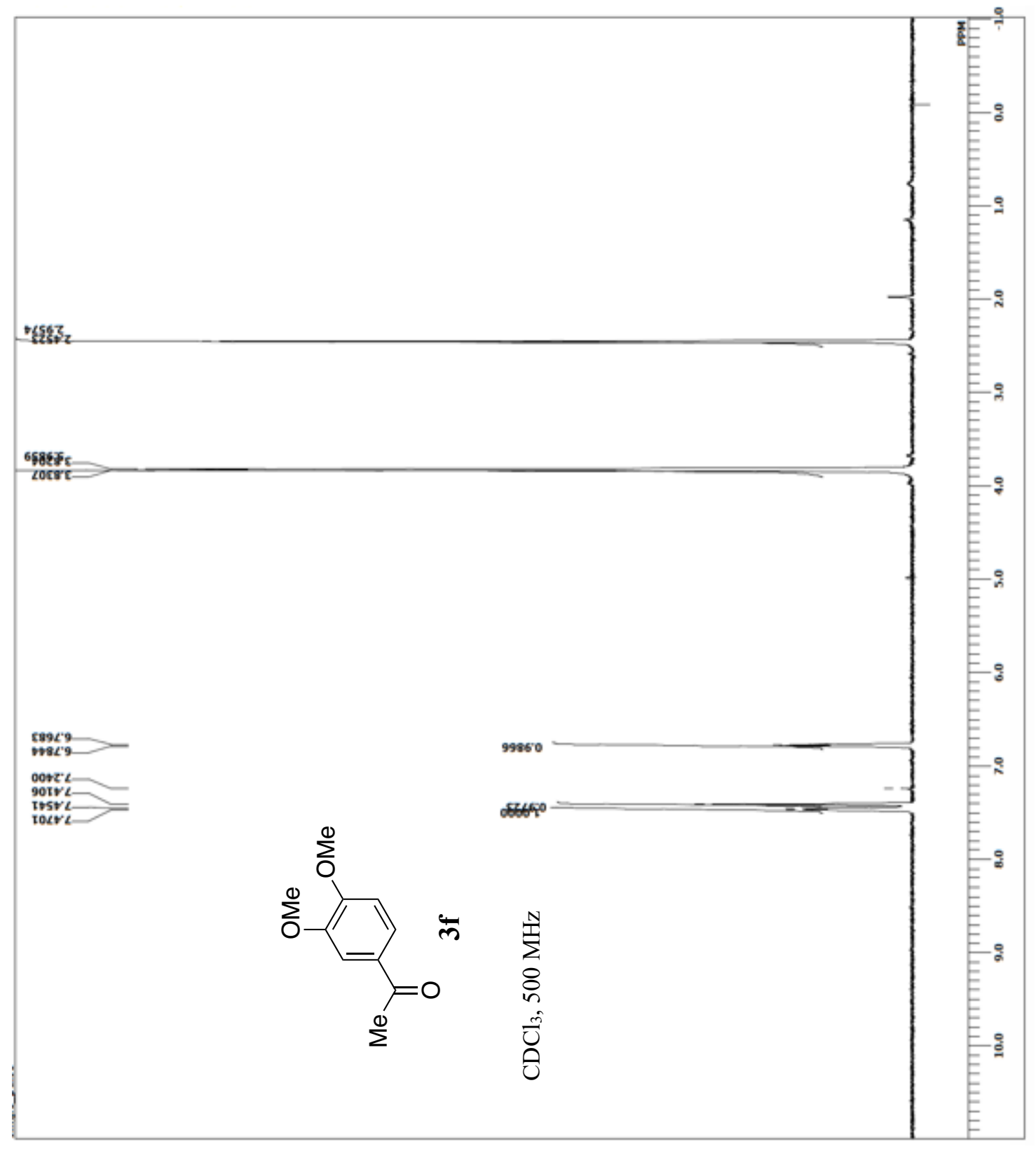




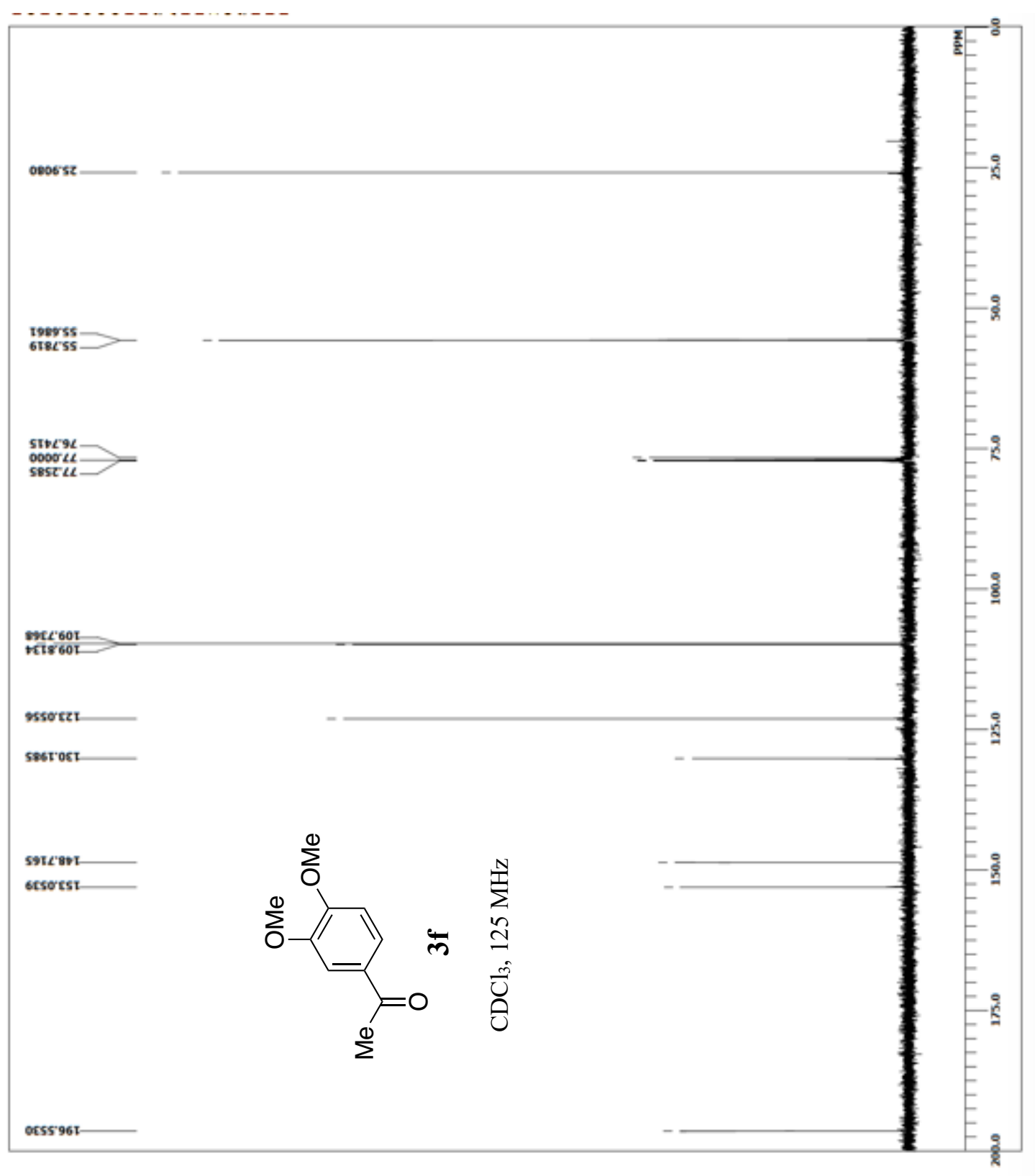




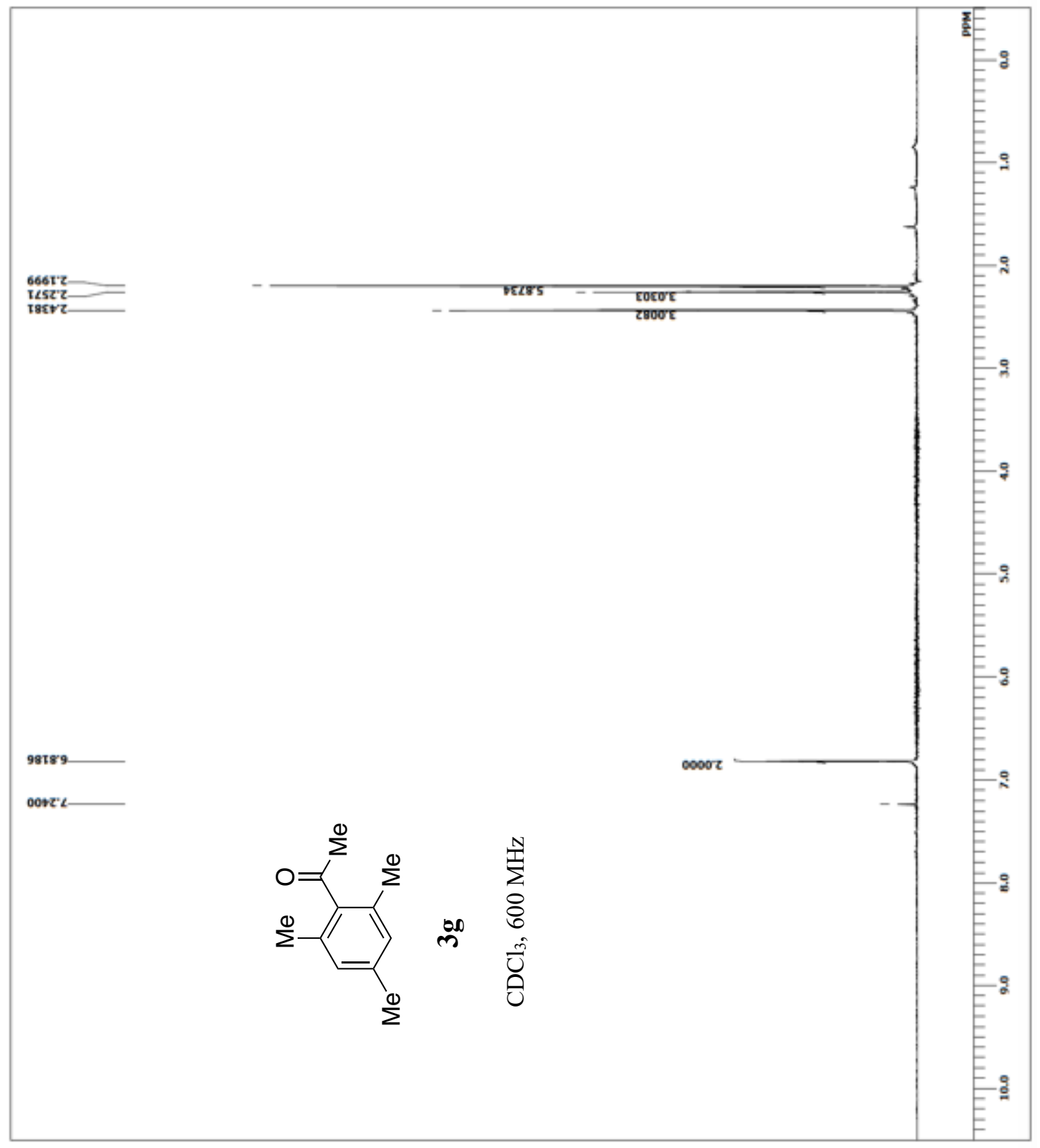




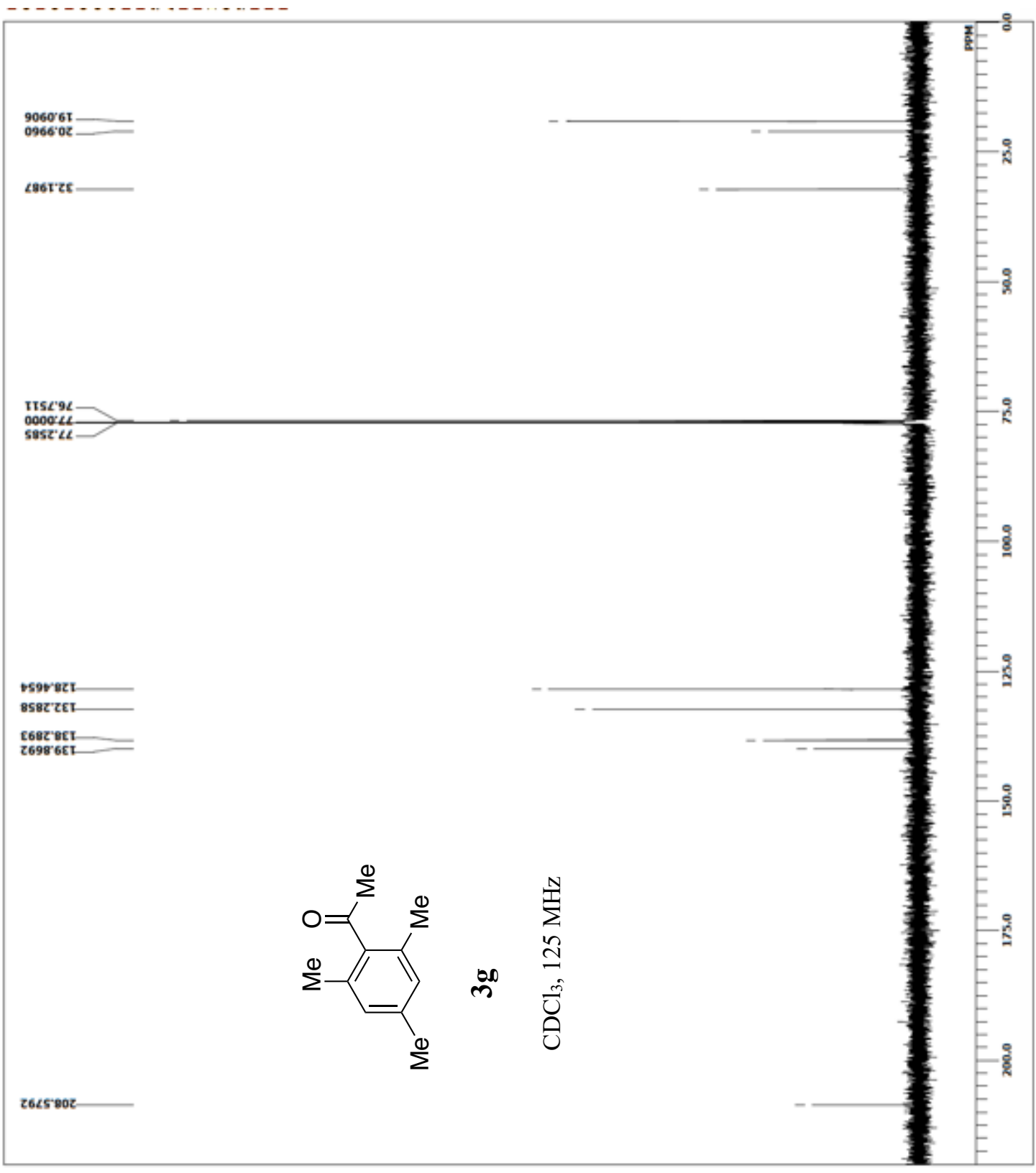









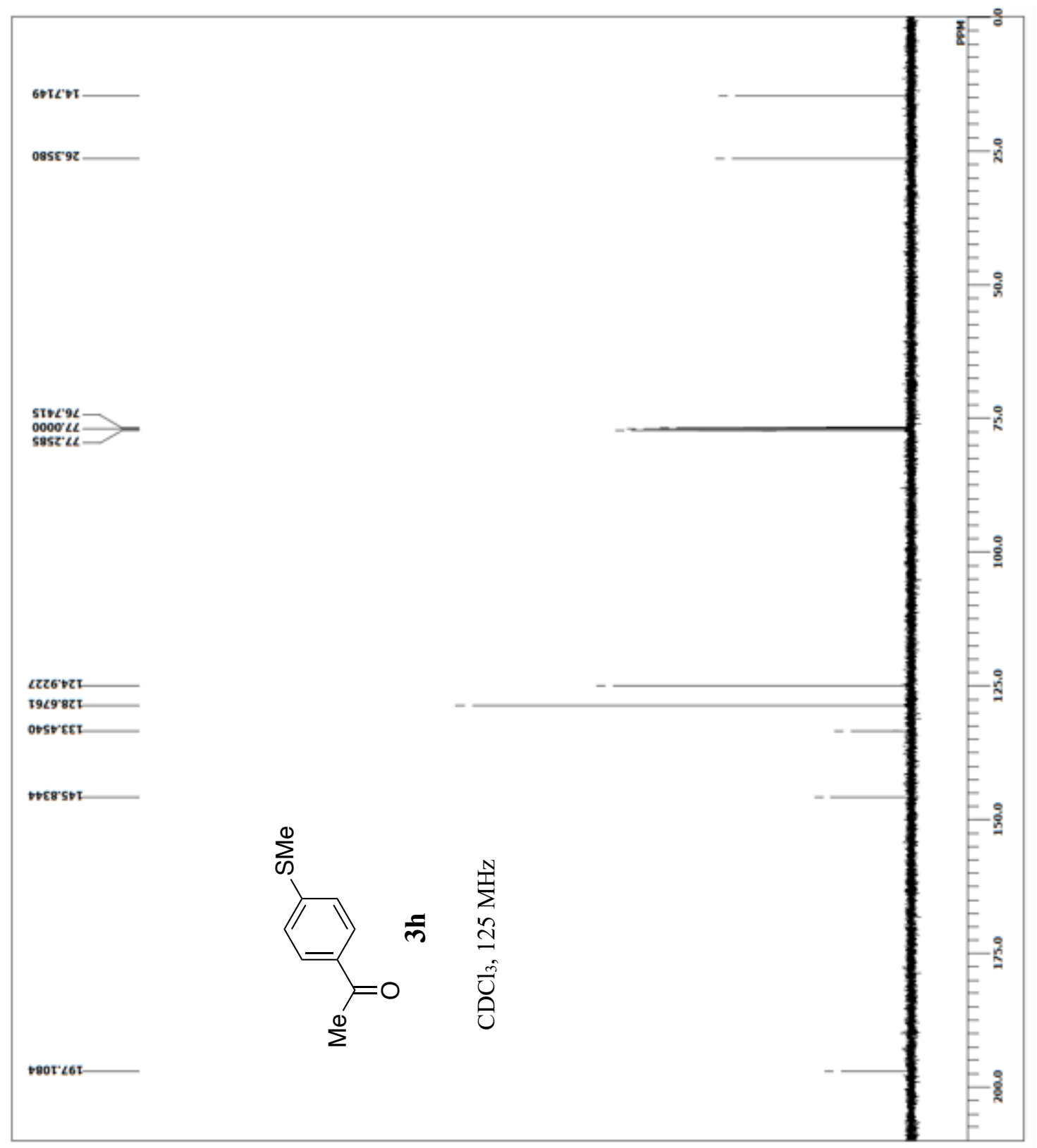




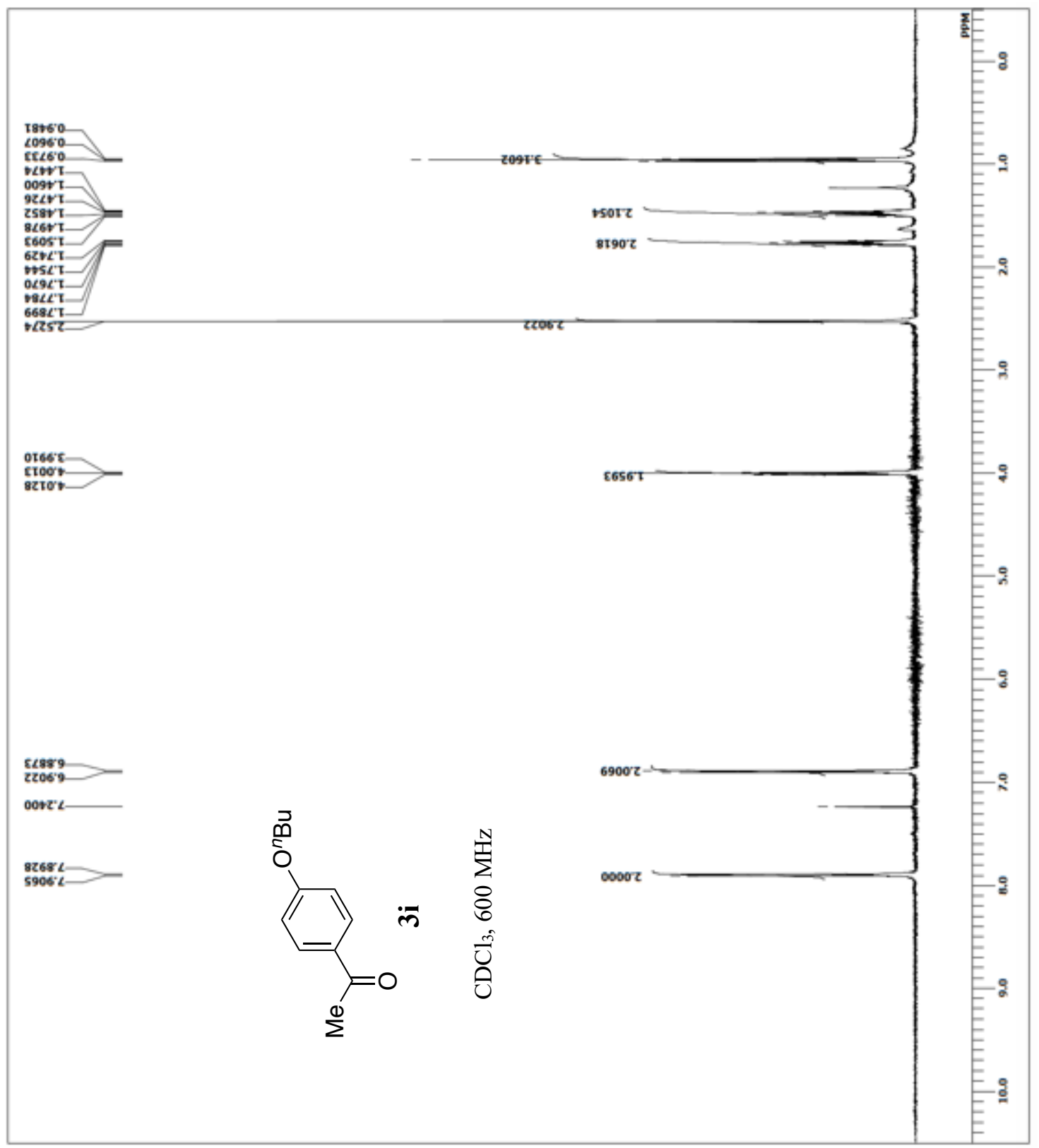









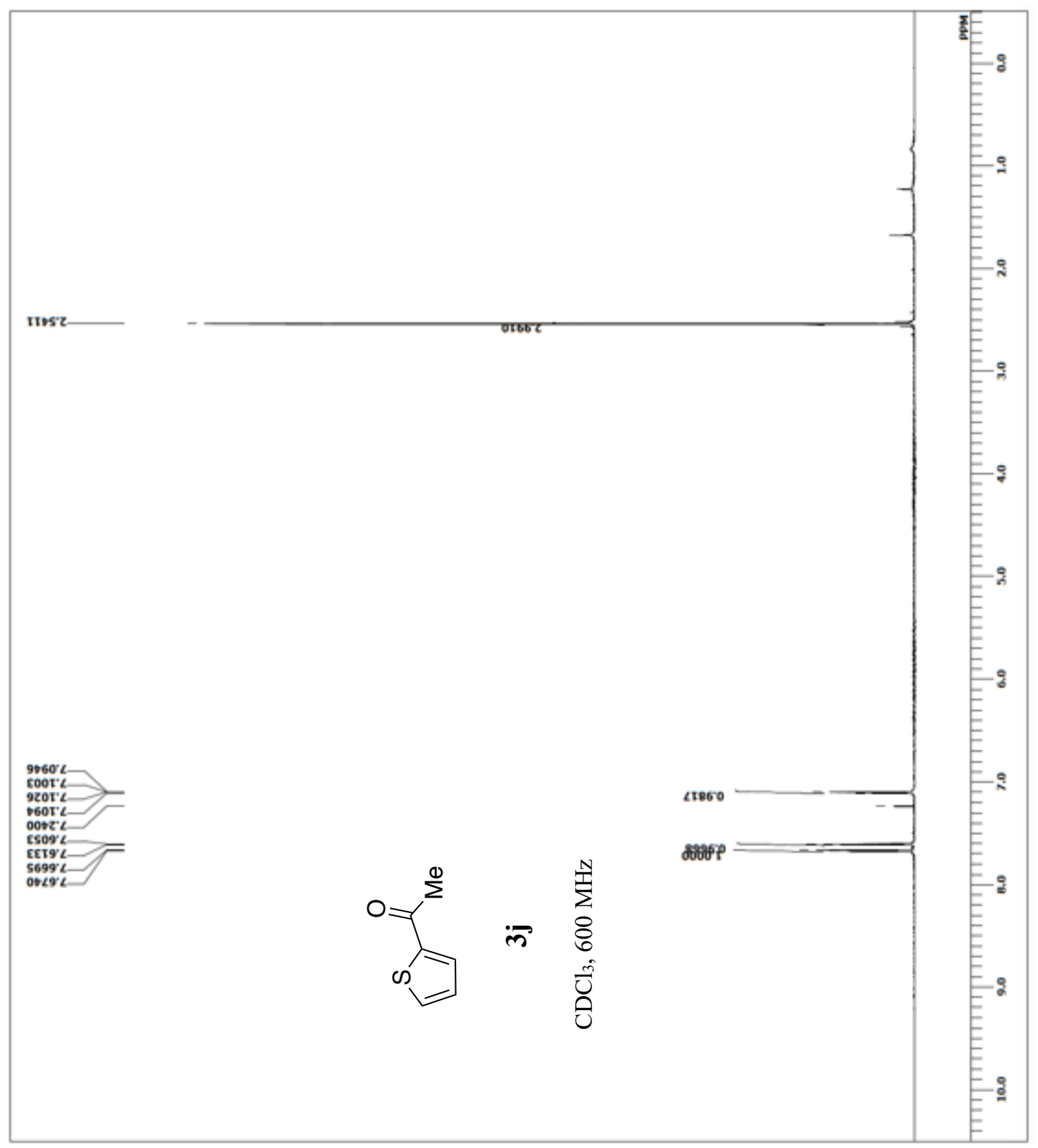




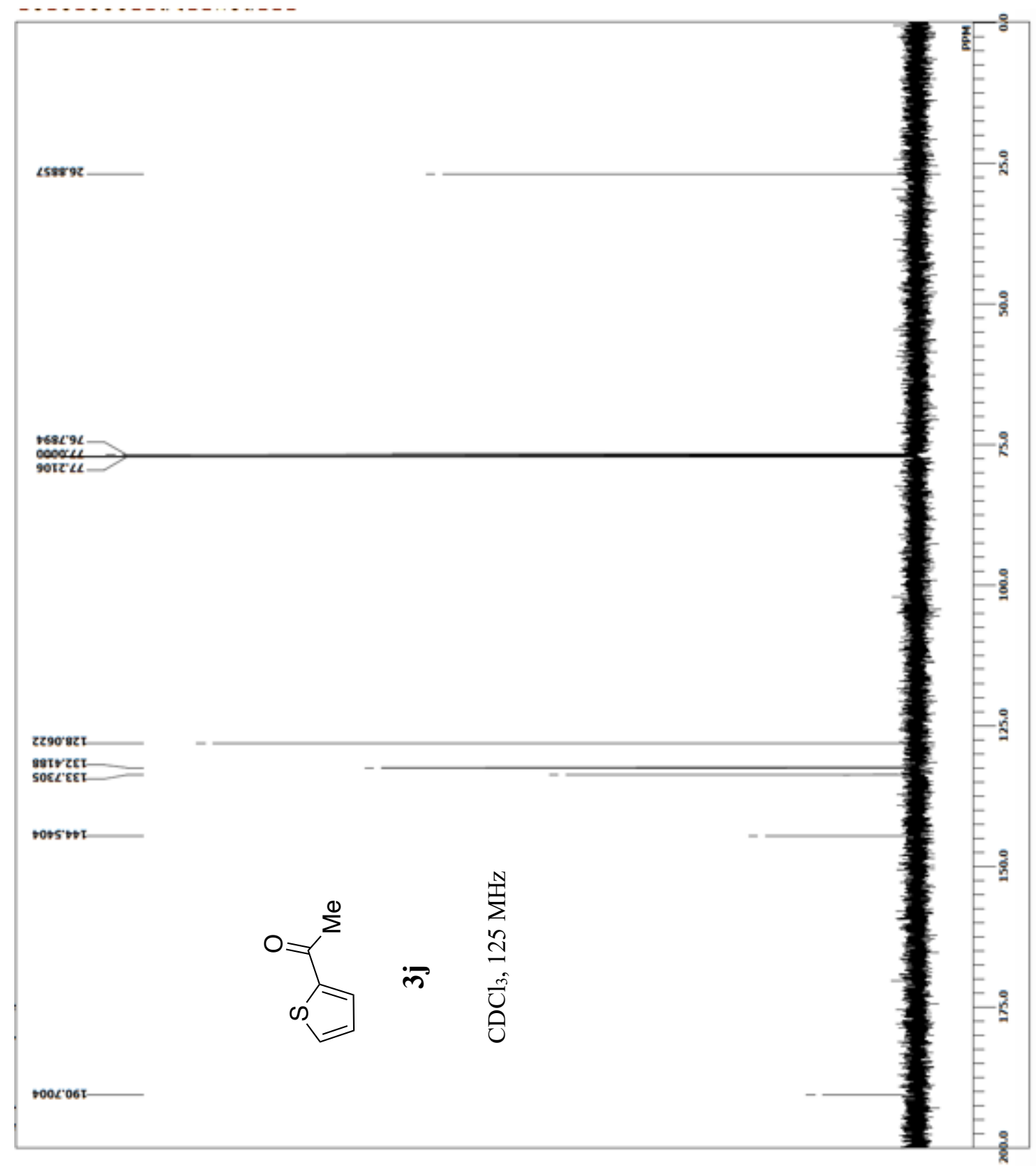

